\title{
Wave turbulence in shallow water models
}

\author{
P. Clark di Leoni, P. J. Cobelli, and P. D. Mininni \\ Departamento de Física, Facultad de Ciencias Exactas y Naturales, Universidad de Buenos Aires and IFIBA, CONICET, \\ Cuidad Universitaria, Buenos Aires 1428, Argentina
}

(Received 16 September 2013; revised manuscript received 17 March 2014; published 30 June 2014)

\begin{abstract}
We study wave turbulence in shallow water flows in numerical simulations using two different approximations: the shallow water model and the Boussinesq model with weak dispersion. The equations for both models were solved using periodic grids with up to $2048^{2}$ points. In all simulations, the Froude number varies between 0.015 and 0.05 , while the Reynolds number and level of dispersion are varied in a broader range to span different regimes. In all cases, most of the energy in the system remains in the waves, even after integrating the system for very long times. For shallow flows, nonlinear waves are nondispersive and the spectrum of potential energy is compatible with $\sim k^{-2}$ scaling. For deeper (Boussinesq) flows, the nonlinear dispersion relation as directly measured from the wave and frequency spectrum (calculated independently) shows signatures of dispersion, and the spectrum of potential energy is compatible with predictions of weak turbulence theory, $\sim k^{-4 / 3}$. In this latter case, the nonlinear dispersion relation differs from the linear one and has two branches, which we explain with a simple qualitative argument. Finally, we study probability density functions of the surface height and find that in all cases the distributions are asymmetric. The probability density function can be approximated by a skewed normal distribution as well as by a Tayfun distribution.
\end{abstract}

DOI: 10.1103/PhysRevE.89.063025

PACS number(s): 47.35.Bb, 47.27.-i, 92.10.Hm, 92.10.Lq

\section{INTRODUCTION}

Turbulence and nonlinear wave interactions in the ocean surface are related to important processes in atmospheric sciences and oceanography, such as the exchange of energy between the atmosphere and the ocean $[1,2]$. This exchange, in turn, plays an important role in the dynamics of the planetary and oceanic boundary layers, with consequences on the transport and mixing of momentum, $\mathrm{CO}_{2}$, and heat [3]. The incorrect modeling of these phenomena affects climate evolution predictions $[4,5]$. Ocean surface waves are also of interest in the search for renewable energies [6].

There are several ocean surface models which provide an excellent framework for studying weak turbulence theory [7-10]. This theory was developed to describe the out-ofequilibrium behavior of systems of dispersive and weakly nonlinear waves (see, e.g., [11,12]). Unlike theories of strong turbulence, for waves and under the assumption of weak nonlinearities, the equations for two-point correlations can be closed and exact solutions with constant flux can be found. Besides this assumption, it is also assumed that wave fields are homogeneous, and that free waves are uncorrelated.

Weak turbulence theory has been applied to capillary and gravito-capillary waves [10], vibrations on a plate [13], rotating flows [14], and magnetohydrodynamic waves $[12,15,16]$. For some of these systems, the predictions of the theory are compatible with results obtained from experiments or from numerical simulations. For example, see Refs. [17-19] for capillary waves, [20] for gravitocapillary waves, [21-23] for vibrations on a plate, and [24,25] for magnetohydrodynamic waves. Although agreement has been found between theory, numerical simulations, and experiments, there are also discrepancies. In some of these cases the compatibility is limited to the spectrum of certain fields (see, e.g., [25]), or to specific configurations used to generate the excitations. Moreover, for many systems it is also not clear whether the weak turbulence approximation holds for all times, as the solutions are not homogeneous in wave number space and at sufficiently small scales eddies may become faster than waves resulting in strong turbulence [26].

One of the most important applications of weak turbulence is in surface gravity waves. In oceanography, the Phillips' spectrum [27], derived using dimensional arguments from strong turbulence and considering the coupling between waves, was long considered to be correct. However, different observational and experimental data [28,29], as well as numerical simulations [30], suggest that the actual spectrum is closer to that of weak turbulence. In fact, Phillips himself suggested that his spectrum may not be valid in the ocean [31]. Nonetheless, a scaling compatible with Phillips' spectrum is still observed in numerical simulations [32] when the forcing is strong. This suggests that while weak turbulence provides an elegant theoretical way to study wave turbulence in the ocean, more considerations are necessary to appropriately describe the diversity of regimes found in these flows [11].

Most of the work done in ocean surface waves under the weak turbulence approximation concerns deep water flows. But the theory can also be applied to the shallow water case, i.e., for gravity waves whose wavelengths are large compared to the height of the fluid column at rest (see [33,34]). In this case, the theory leads to the prediction that the energy spectrum follows a $\sim k^{-4 / 3}$ behavior. Behavior compatible with this prediction was found both experimentally and observationally [35-37]. It was also found that an inertial range with a $\sim k^{-2}$ dependency can develop in the shallower regions of the fluid. The comparison between different shallow water models, with different degrees of shallowness (and of dispersion) is therefore of interest, e.g., for the study of waves in basins with inhomogeneous depth.

In the shallow water regime there are several models that can be considered to describe the ocean surface. There is the linear theory (see, e.g., [38]) which can predict the dispersion relation of small amplitude waves, but which is insufficient to study turbulence. There are also nonlinear theories, such as 
the shallow water model [39] for nondispersive waves, as well as the Boussinesq model [40] for weakly dispersive waves which is the one used in some of the most recent works on the subject [34]. While the former nonlinear model is valid in the strict shallow water limit, the latter can be used in cases in which the wavelengths are closer to (albeit still larger than) the depth of the basin.

In the present work, we study turbulent solutions of the shallow water model and of the Boussinesq equations using direct numerical simulations. Previous numerical studies considered the Hamiltonian equations for a potential flow with a truncated nonlinear term, or the kinetic equations resulting from weak turbulence theory at moderate spatial resolution [32,41] (with the notable exception of [42]). Here, we solve the primitive equations, without truncating the nonlinear terms, potentially allowing for the development of vortical motions and of strong interactions between waves, and with spatial resolutions up to $2048^{2}$ grid points.

The paper is organized as follows. In Sec. II we introduce both models, show the assumptions made in order to obtain them, derive their energy balance equations, and briefly discuss the predictions obtained in the framework of weak turbulence theory. In Sec. III we describe the numerical methods employed and the simulations. Then, in Sec. IV we introduce several dimensionless numbers defined to characterize the flows, and present the numerical analysis and results. We present wave number energy spectra and fluxes, time-resolved spectra (as a function of the wave number and frequency), frequency spectra, and probability density functions of the fluid free surface height. Finally, in Sec. V we present the conclusions. The most important results are: (a) As in previous experimental and observational studies $[35,36]$ we find now in simulations that different regimes arise depending on the fluid depth and the degree of nonlinearity of the system. (b) We obtain a power spectrum of the surface height compatible (within statistical uncertainties) with $\sim k^{-2}$ in the shallow water (nondispersive) case, and one compatible with a $\sim k^{-4 / 3}$ spectrum as the fluid depth is increased using the Boussinesq (weakly dispersive) model. The latter spectrum is also compatible with predictions of weak turbulence theory [34]. (c) Dispersion in the Boussinesq model results in more prominent small-scale features and the development of rapidly varying waves. (d) In the weakly dispersive regime, the nonlinear dispersion relation obtained from the simulations has two branches in a range of wave numbers, one branch corresponding to nondispersive waves, and another corresponding to dispersive waves. We interpret this as the result of short wavelength waves seeing an effectively deeper flow resulting from the interaction with waves with very long wavelength. (e) The probability density function of surface height can be approximated by both skewed normal and Tayfun distributions. In the latter case, the parameters of the distribution are compatible with those previously found in observations and experiments [37].

\section{THE SHALLOW WATER AND BOUSSINESQ EQUATIONS}

Let us consider a volume of an incompressible fluid with uniform and constant (in time) density, with its bottom surface in contact with a flat and rigid boundary, and free surface at

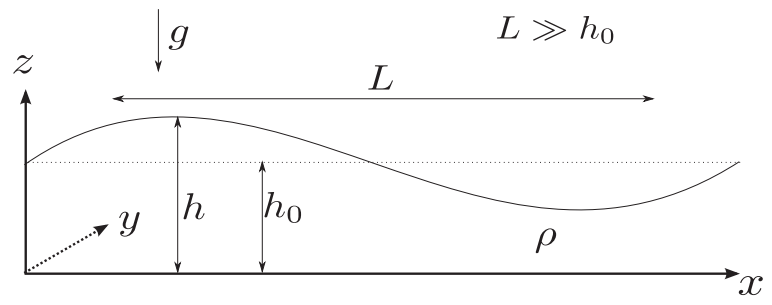

FIG. 1. The shallow water geometry considered in the simulations: $x$ and $y$ are the horizontal coordinates, and $z$ is the vertical coordinate. The surface height is $h$, with $h_{0}$ the height of the fluid column at rest. The fluid surface is at pressure $p_{0} . L$ is a characteristic horizontal length (assumed to be much larger than $h_{0}$ ). Gravity acts on the $-\hat{z}$ direction and its acceleration has a value of $g$.

pressure $p_{0}$. A sketch illustrating the configuration is shown in Fig. 1: $x$ and $y$ are the horizontal coordinates, $z$ is the vertical one, $h$ is the height of the fluid column (i.e., the $z$ value at the free surface), $h_{0}$ is the height of the fluid column at rest, $L$ is a characteristic horizontal length, gravity acts on the $-\hat{z}$ direction, and its value is given by $g$. It is assumed that $L \gg h_{0}$ as we are interested in shallow flows.

In the inviscid case, both the Euler equation and the incompressibility condition hold in the fluid body,

$$
\begin{gathered}
\frac{\partial \mathbf{v}}{\partial t}+(\mathbf{v} \cdot \nabla) \mathbf{v}=-\frac{1}{\rho} \nabla p-g \hat{z}, \\
\nabla \cdot \mathbf{v}=0 .
\end{gathered}
$$

Under certain assumptions, to be discussed in the following paragraphs, the evolution of the free surface can be adequately described by means of a vector equation for the two horizontal components of the velocity at the interface, plus an equation for the local height of the fluid column.

\section{A. Linear dispersion relation}

Considering the case of very small amplitude waves, one can linearize the system of Eqs. (1) and (2) (see, e.g., [38]). The solutions of the resulting equations are gravity waves with the following dispersion relation,

$$
\omega^{2}=g k \frac{1-e^{-2 k h_{0}}}{1+e^{-2 k h_{0}}} .
$$

We are interested in the shallow water case, i.e., when $h_{0} \ll$ $L \Rightarrow h_{0} k \ll 1$. In that limit the following dispersion relation results:

$$
\omega=\sqrt{g h_{0}} k=c_{0} k,
$$

where $c_{0}=\sqrt{g h_{0}}$ is the phase velocity. Note in this case waves are not dispersive, unlike the general case given by Eq. (3).

\section{B. Shallow water model}

It is possible to derive a set of nonlinear equations for the surface height and the horizontal velocity at the surface by using the fact that the fluid layer is shallow. Considering the characteristic magnitudes of all quantities in Eq. (1) $\left(L, p_{0}\right.$, $h_{0}, g$, etc.), and using the fact that in a shallow flow $h_{0} k \ll 1$ with $k=2 \pi / L$, one obtains hydrostatic balance in the vertical 
direction (for further details, see [39]), which results in the pressure profile,

$$
p=\rho g(h-z)+p_{0} .
$$

As $h$ is not a function of $z$, neither will be the horizontal pressure gradient and the horizontal components of the velocity (as long as they do not depend initially on $z$ ). In this way, the horizontal components of Eq. (1) can be written as

$$
\frac{\partial \mathbf{u}}{\partial t}=-(\mathbf{u} \cdot \nabla) \mathbf{u}-g \nabla h
$$

where $\mathbf{u}(x, y, t)=v_{x} \hat{x}+v_{y} \hat{y}$ is the horizontal velocity, and $\nabla$ is now the horizontal gradient.

Using the fact that $v_{x}$ and $v_{y}$ are independent of $z$ we can integrate the incompressibility condition, obtaining

$$
v_{z}(x, y, z, t)=-z\left(\frac{\partial v_{x}}{\partial x}+\frac{\partial v_{y}}{\partial y}\right)+\tilde{v}_{z}(x, y, t) .
$$

Finally, by taking the appropriate boundary conditions and setting $z=h(x, y, t)$, Eq. (7) provides an equation for the evolution of the height of the fluid column, namely

$$
\frac{\partial h}{\partial t}+\frac{\partial}{\partial x}\left(h v_{x}\right)+\frac{\partial}{\partial y}\left(h v_{y}\right)=0 .
$$

Note that we do not have to assume irrotationality to derive either Eq. (6) or Eq. (8).

If we linearize these equations, we find again the dispersion relation given by Eq. (4), as expected. In the presence of external forcing $\mathbf{F}$, and viscosity $v$, the equations can be written as

$$
\begin{gathered}
\frac{\partial \mathbf{u}}{\partial t}=-(\mathbf{u} \cdot \nabla) \mathbf{u}-g \nabla h+\frac{v}{h} \nabla \cdot(h \nabla \mathbf{u})+\mathbf{F}, \\
\frac{\partial h}{\partial t}=-\nabla \cdot(h \mathbf{u}) .
\end{gathered}
$$

We will refer to this set of equations as the shallow water model, or SW model. In these equations the viscosity $v$ was added to the horizontal velocity field $\mathbf{u}$, which behaves as a compressible flow (i.e., it has nonzero divergence, see [43]). This choice of the viscous term ensures conservation of the momentum $h \mathbf{u}$, and also that energy dissipation is always negative, as in Sec. II D.

\section{Boussinesq model}

As the depth of the fluid increases, dispersion becomes important. There are several models that introduce weak dispersive effects perturbatively, but many are built to study waves propagating in only one direction. The Boussinesq equations for surface waves (see, e.g., $[40,44]$ ) provide a model to study weakly dispersive waves propagating in any direction. This model not only broadens the range of physical phenomena encompassed by the SW model, but adding dispersive effects also makes it more enticing to weak turbulence theory, for which dispersion effects are of the utmost importance.

Let us take a look at the first terms in the Taylor expansion of the dispersion relation in Eq. (3),

$$
\omega^{2}=c_{0}^{2} k^{2}-\frac{1}{3} c_{0}^{2} h_{0}^{2} k^{4}+\ldots,
$$

where the first term is the nondispersive shallow water term. The idea is to add terms to Eqs. (9) and (10) such that the linear dispersion relation of the new system coincides, up to the fourth order, with the expansion in Eq. (11). This can be done by adding the term $h_{0}^{2} \nabla^{2} \partial_{t} \mathbf{u} / 3$ to Eq. (9), resulting in the following system,

$$
\frac{\partial \mathbf{u}}{\partial t}=-(\mathbf{u} \cdot \nabla) \mathbf{u}-g \boldsymbol{\nabla} h+\frac{1}{3} h_{0}^{2} \nabla^{2} \frac{\partial \mathbf{u}}{\partial t}+\frac{v}{h} \nabla \cdot(h \nabla \mathbf{u})+\mathbf{F},
$$

$$
\frac{\partial h}{\partial t}=-\nabla \cdot(h \mathbf{u})
$$

We will refer to this system as the Boussinesq model, or BQ model. For $\mathbf{F}=0$ and $v=0$, the dispersion relation obtained by linearizing these equations is

$$
\omega=\frac{c_{0} k}{\sqrt{1+\frac{h_{0}^{2} k^{2}}{3}}},
$$

which, up to the fourth order, coincides with Eq. (3).

Note that there are other choices for the extra term in Eq. (9) that result in many formulations of the Boussinesq model, all compatible up to fourth order in a Taylor expansion in terms of $h_{0} k$ [40]. The formulation we use here was employed in previous studies of wave turbulence [34], and is also easy to solve numerically using pseudospectral methods by writing Eq. (12) as

$$
\frac{\partial \mathbf{u}^{\prime}}{\partial t}=-(\mathbf{u} \cdot \nabla) \mathbf{u}-g \nabla h+\frac{v}{h} \nabla \cdot(h \nabla \mathbf{u})+\mathbf{F},
$$

where $\mathbf{u}^{\prime}=\mathcal{H} \mathbf{u}$, and where $\mathcal{H}=\left(1-h_{0}^{2} \nabla^{2} / 3\right)$ is the Helmholtz operator. This operator can be easily inverted in Fourier space $[45,46]$, and the resulting equations can be efficiently solved by means of pseudospectral codes. It is interesting that the same operator appears in Lagrangianaveraged models [47]. In these models, and in regularized versions of the shallow water equations [48], it introduces dispersion that results in an accumulation of energy at small scales [49].

\section{Energy balance}

An exact energy balance can be easily derived for the SW model. The equation is useful to verify conservation in pseudospectral codes. By taking the dot product of Eq. (9) and $h \mathbf{u}$, setting $\mathbf{F}=0$, and using Eq. (10), we obtain

$$
\begin{aligned}
\frac{\partial}{\partial t}\left(\frac{h u^{2}}{2}+g \frac{h^{2}}{2}\right)= & -\nabla \cdot\left(\frac{h u^{2}}{2} \mathbf{u}+g h^{2} \mathbf{u}\right) \\
& +v \mathbf{u} \cdot[\nabla \cdot(h \nabla \mathbf{u})] .
\end{aligned}
$$

Integrating in $x$ and $y$ over an area $A$ and taking periodic boundary conditions yields

$$
\frac{d E}{d t}=-2 v Z
$$

where

$$
E=\frac{1}{A} \iint\left(\frac{h u^{2}}{2}+g \frac{h^{2}}{2}\right) d x d y
$$


is the mean total energy, and

$$
Z=\frac{1}{A} \iint \frac{h|\nabla \mathbf{u}|^{2}}{2} d x d y
$$

is a mean pseudoenstrophy, such that $-2 v Z$ is the mean energy dissipation rate. As $h$ is always positive, the energy dissipation is always negative. The total energy is conserved when $v=0$.

Now we can define

$$
U=\frac{1}{A} \iint \frac{h u^{2}}{2} d x d y
$$

as the mean kinetic energy, and

$$
V=\frac{1}{A} \iint g \frac{h^{2}}{2} d x d y
$$

as the mean potential energy, such that the sum of both gives the mean total energy $E$.

The dispersive term present in the BQ model changes the balance given by Eq. (17). However, since the extra term is of order $\left(h_{0} / L\right)^{2}$, as long as we are in a sufficiently shallow flow it will be very small, and therefore, negligible for the conservation of energy. We verified this is the case in our numerical simulations.

\section{E. Weak turbulence prediction}

We briefly present some results obtained in the framework of weak turbulence theory for the BQ model (as the derivation is a bit cumbersome, only a general outline will be given here; please see [34] for details). Weak turbulence is studied in the BQ model assuming the fluid is inviscid and irrotational, so that the velocity can be written in terms of a velocity potential. To obtain a statistical description of the wave field, it is also assumed that it is homogeneous and that the free modes are uncorrelated.

At first sight, the quadratic nonlinear terms in Eqs. (12) and (13) indicate modes interact in triads, with the wave vectors of the three interacting modes lying over a triangle, and the three frequencies satisfying the resonant condition (see, e.g., [12]):

$$
\begin{gathered}
\mathbf{k}=\mathbf{p}+\mathbf{q}, \\
\omega(\mathbf{k})=\omega(\mathbf{p})+\omega(\mathbf{q}) .
\end{gathered}
$$

However, as there are no three wave vectors $\mathbf{k}, \mathbf{p}, \mathbf{q}$ that satisfy these two conditions when the dispersion relation is given by Eq. (11), three wave interactions are forbidden. Thus, only four wave interactions are present (which do satisfy their corresponding condition).

After a transformation of the fields, it is possible to write an equation for the evolution of the two-point correlator of the transformed fields. This is the so-called kinetic equation, and has the following form:

$$
\begin{aligned}
\frac{\partial N_{0}}{\partial t}= & 4 \pi \int\left|T_{0,1,2,3}\right|^{2} N_{0} N_{1} N_{2} N_{3} \\
& \times\left(\frac{1}{N_{0}}+\frac{1}{N_{1}}-\frac{1}{N_{2}}-\frac{1}{N_{3}}\right), \\
& \times \delta\left(\mathbf{k}_{0}+\mathbf{k}_{0}-\mathbf{k}_{2}-\mathbf{k}_{3}\right), \\
& \times \delta\left(\omega_{0}+\omega_{0}-\omega_{2}-\omega_{3}\right) d \mathbf{k}_{123},
\end{aligned}
$$

where $N_{i}=N\left(\mathbf{k}_{i}\right)$ is the wave action spectral density (i.e., the two-point correlator of the wave action, the latter being a quantity proportional to the surface height), the deltas express the fact that interactions are between four wave vectors and their associated frequencies, $T_{0,1,2,3}$ is the coupling coefficient between the four modes, and $d \mathbf{k}_{123}=d \mathbf{k}_{1} d \mathbf{k}_{2} d \mathbf{k}_{3}$. From this equation, dimensional analysis yields the following expression for the energy spectrum,

$$
E(k) \sim k^{-4 / 3}
$$

From this spectrum and using dimensional analysis, it is easy to show that in the presence of dissipation, the dissipation wave number in such a flow is $k_{\eta} \sim\left[\epsilon /\left(h_{0}^{2} \nu^{3}\right)\right]^{1 / 5}$, where $\epsilon$ is the mean energy injection rate.

A scaling compatible with an $\sim k^{-4 / 3}$ spectrum was observed in laboratory and field data sets $[35,36]$, where a spectrum compatible with $\sim k^{-2}$ was also found in shallower regions of the fluid.

The prediction in Eq. (25) applies to the BQ model, when dispersion is not negligible. Before proceeding, we should comment on some peculiarities of the SW model regarding wave turbulence. First, an inspection of its dispersion relation, Eq. (4), indicates that three wave interactions are possible in this model, and as a result the arguments above for four-wave interactions do not apply. Weak turbulence theory can be used in systems with three-wave interactions (with the case of deep water flows being a paradigmatic one, but see also the case of rotating [14] and of magnetohydronamic [15] flows). However, the SW model is nondispersive, and as a result the resonance condition is only satisfied for collinear wave vectors. Resonant interactions can then only couple modes that propagate in the same direction (i.e., along the ray of the wave), and nonresonant interactions must be taken into account to consider other couplings. But more importantly, dispersion is crucial in weak turbulence theory to have decorrelation between different waves: Without dispersion, all modes propagate with the same velocity, and the modes initially correlated remain correlated for all times (see, e.g., [50] for a discussion of these effects in the context of acoustic turbulence).

\section{NUMERICAL SIMULATIONS}

We performed several numerical simulations of both the shallow water and the Boussinesq models. These were done using the GHOST code [51-53], which uses a pseudospectral method with periodic boundary conditions on a $L_{0} \times L_{0}=$ $2 \pi \times 2 \pi$ sized box (with $L_{0}$ the box length), the " $2 / 3$ rule" for the dealiasing [54], explicit second-order Runge-Kutta for time stepping, and is parallelized using MPI and OPENMP. Almost all simulations shown here were done on grids of $N^{2}=2058^{2}$ points, with a few on grids of $N^{2}=1024^{2}$ or $512^{2}$ points (with $N$ the linear resolution). As a result of dealiasing, the maximum resolved wave number is

$$
k_{\max }=N / 3 .
$$

Note all magnitudes in the code are dimensionless, with the smallest wave number $k_{\min }=2 \pi / L_{0}=1$, and the largest wave number $k_{\max }=2 \pi / \lambda_{\min }$ being associated with the minimum resolved scale $\lambda_{\min }$. 
All runs are direct numerical simulations, with all relevant space and time scales resolved explicitly. The pseudospectral method with the $2 / 3$ rule is equivalent to a purely spectral method [54]: It converges exponentially fast, it conserves all quadratic invariants of the equations (i.e., there is no numerical dissipation introduced by the method), and it also has no numerical dispersion. All this was verified explicitly during the development of the code, using several test problems for the SW and BQ equations.

Most previous numerical studies on wave turbulence in gravity waves were done at lower resolutions, with the exception of [42]. But the key difference between previous simulations and the ones presented here (besides the fact that these are for shallow flows, not for deep flows) is that the physical model we use does not assume potential flow, and, more importantly, we do not truncate the nonlinear term, thus retaining all high order nonlinearities. Another difference is that we do not introduce an artificial dissipation term as it is usually done, but one based on physical grounds. The key motivation for these choices is to be able to compare with experiments in the future, where vortical motions can develop, and where dissipation also plays a non-negligible role. To achieve higher resolutions than the ones studied here becomes increasingly more expensive as the BQ model is dispersive.

All the simulations were started from the fluid at rest. An external mechanical forcing injected energy in the system, allowing it to reach for sufficiently long times an out-ofequilibrium turbulent steady state, after an initial transient. To excite waves, and prevent external injection of energy into vortical motions, the forcing had the following form:

$$
\mathbf{F}=\nabla f
$$

where $f$ is a randomly generated scalar function, with a time correlation of one time unit, amplitude $f_{0}$, and applied in a band of wave numbers in Fourier space between $k_{f_{1}}$ and $k_{f_{2}}$ (see Tables I, II, and III). Note that having a mechanical forcing in the momentum equation adds an extra term to the right-hand side of Eq. (17),

$$
\frac{d E}{d t}=-2 \nu Z+\epsilon,
$$

where the mean energy injection rate can be computed as

$$
\epsilon=\frac{1}{A} \iint_{A} h \mathbf{u} \cdot \mathbf{f} d x d y .
$$

Under the procedure described above, the typical evolution of the energy in a numerical simulation is shown in Fig. 2. The energy starts from the value corresponding to the fluid at rest (i.e., all the energy is the potential energy associated with the equilibrium height $h_{0}$ ). The total energy then grows under the action of the external mechanical forcing, and after $t \approx 80$ the system reaches a turbulent steady state in which the energy fluctuates around a mean value, and in which the energy injection and dissipation are equilibrated on the average. Even though pseudospectral methods are known to introduce no numerical dissipation, in the inset of Fig. 2 we also show explicitly that the energy balance [Eq. (28)] is satisfied with an error of order $10^{-7}$, which remains stable and does not grow even after integrating for very long times.

To ensure that the flow in the simulations remained shallow for all excited wave numbers, we enforced the following condition:

$$
\begin{aligned}
\frac{h_{0}}{\lambda_{\min }} & =h_{0} \frac{k_{\max }}{2 \pi}<1 \\
& \Rightarrow h_{0}<\frac{6 \pi}{N},
\end{aligned}
$$

where $\lambda_{\min }$ is, as already mentioned, the shortest wavelength resolved by the code in virtue of the condition given by Eq. (26).

\section{RESULTS}

\section{A. Description and classification of the simulations}

The spectral behavior of the flow in the simulations depends on the external parameters. We can independently control the height of the fluid at rest $h_{0}$, the viscosity $v$, the gravity acceleration $g$, the amplitude of the forcing $f_{0}$, the range of wave numbers in which the force is applied, and the linear resolution $N$. However, all these parameters can be reduced to a smaller set of dimensionless controlling parameters.

\begin{tabular}{|c|c|c|c|c|c|c|c|c|}
\hline Simulation & $\mathrm{Re}$ & $\mathrm{Fr}$ & $D_{s}$ & $N_{l}$ & $h_{0} / L_{0}$ & $f_{0} / U_{0}$ & {$\left[k_{f_{1}}, k_{f_{2}}\right]$} & $N$ \\
\hline$A 01$ & 260 & 0.005 & 0.27 & $1.2 \times 10^{-5}$ & $8.0 \times 10^{-4}$ & 0.76 & {$[3,8]$} & 1024 \\
\hline$A 02$ & 370 & 0.0059 & 0.22 & $1.6 \times 10^{-5}$ & $6.4 \times 10^{-4}$ & 0.71 & {$[3,8]$} & 1024 \\
\hline$A 03$ & 820 & 0.0075 & 0.33 & $2.6 \times 10^{-5}$ & $4.9 \times 10^{-4}$ & 0.64 & {$[3,8]$} & 2048 \\
\hline$A 04$ & 760 & 0.0067 & 0.36 & $2.2 \times 10^{-5}$ & $5.3 \times 10^{-4}$ & 0.69 & {$[3,8]$} & 2048 \\
\hline$A 05$ & 760 & 0.007 & 0.33 & $2.3 \times 10^{-5}$ & $4.8 \times 10^{-4}$ & 0.69 & {$[3,8]$} & 2048 \\
\hline$A 06$ & 360 & 0.0066 & 0.33 & $2.1 \times 10^{-5}$ & $4.8 \times 10^{-4}$ & 0.73 & {$[3,8]$} & 2048 \\
\hline$A 07$ & 570 & 0.0091 & 0.43 & $4.1 \times 10^{-5}$ & $6.4 \times 10^{-4}$ & 0.92 & {$[3,8]$} & 2048 \\
\hline$A 08$ & 350 & 0.0083 & 0.43 & $3.4 \times 10^{-5}$ & $6.4 \times 10^{-4}$ & 1 & {$[3,8]$} & 2048 \\
\hline$A 09$ & 290 & 0.0086 & 0.43 & $3.6 \times 10^{-5}$ & $6.4 \times 10^{-4}$ & 0.98 & {$[3,8]$} & 2048 \\
\hline$A 10$ & 420 & 0.012 & 0.43 & $7.8 \times 10^{-5}$ & $6.4 \times 10^{-4}$ & 1.4 & {$[3,8]$} & 2048 \\
\hline
\end{tabular}

TABLE I. Dimensionless numbers (defined in the text) and parameters for runs in set $A$. Re is the Reynolds number, Fr is the Froude number, $D_{s}$ is the dispersivity, $N_{l}$ is the nonlinear number, $h_{0} / L_{0}$ is the height of the fluid at rest divided by the length of the box, $f_{0} / U_{0}$ the amplitude of the forcing divided by the rms speed, $k_{f_{1}}$ and $k_{f_{2}}$ are, respectively, the minimum and maximum wave numbers in which the random forcing is applied, and $N$ is the linear resolution. In all cases, the Boussinesq model was solved. 
TABLE II. Dimensionless numbers and parameters for runs in set $B$. Labels are as in Table I. The Boussinesq model was solved in all cases except for runs $B_{\mathrm{SW}} 11$ and $B_{\mathrm{SW}} 12$, that were done solving the shallow water model.

\begin{tabular}{|c|c|c|c|c|c|c|c|c|}
\hline Simulation & $\mathrm{Re}$ & $\mathrm{Fr}$ & $D_{s}$ & $N_{l}$ & $h_{0} / L_{0}$ & $f_{0} / U_{0}$ & {$\left[k_{f_{1}}, k_{f_{2}}\right]$} & $N$ \\
\hline$B 01$ & 5600 & 0.022 & 0.14 & $2.5 \times 10^{-4}$ & $8.0 \times 10^{-4}$ & 0.45 & {$[1,5]$} & 512 \\
\hline$B 02$ & 3700 & 0.015 & 0.14 & $1.1 \times 10^{-4}$ & $8.0 \times 10^{-4}$ & 0.34 & {$[1,5]$} & 512 \\
\hline$B 03$ & 5000 & 0.012 & 0.14 & $7.2 \times 10^{-5}$ & $8.0 \times 10^{-4}$ & 0.29 & {$[1,5]$} & 512 \\
\hline B04 & 7100 & 0.013 & 0.11 & $9.1 \times 10^{-5}$ & $3.2 \times 10^{-4}$ & 0.24 & {$[1,5]$} & 1024 \\
\hline B05 & 830 & 0.005 & 0.11 & $1.2 \times 10^{-5}$ & $3.2 \times 10^{-4}$ & 0.8 & {$[3,8]$} & 1024 \\
\hline$B 06$ & 1200 & 0.011 & 0.11 & $6.2 \times 10^{-5}$ & $3.2 \times 10^{-4}$ & 0.57 & {$[3,8]$} & 1024 \\
\hline B07 & 120 & 0.0046 & 0.14 & $1.0 \times 10^{-5}$ & $8.0 \times 10^{-4}$ & 0.82 & {$[3,8]$} & 512 \\
\hline B08 & 980 & 0.012 & 0.17 & $7.9 \times 10^{-5}$ & $2.5 \times 10^{-4}$ & 0.54 & {$[3,8]$} & 2048 \\
\hline$B 09$ & 2500 & 0.038 & 0.27 & $7.4 \times 10^{-4}$ & $8.0 \times 10^{-4}$ & 0.31 & {$[3,8]$} & 1024 \\
\hline$B 10$ & 670 & 0.0042 & 0.17 & $8.5 \times 10^{-6}$ & $2.5 \times 10^{-4}$ & 0.79 & {$[3,8]$} & 2048 \\
\hline$B_{\mathrm{SW}} 11$ & 100 & 0.0039 & 0.14 & $7.6 \times 10^{-6}$ & $8.0 \times 10^{-4}$ & 0.96 & {$[3,8]$} & 512 \\
\hline$B_{\mathrm{SW}} 12$ & 470 & 0.013 & 0.11 & $9.0 \times 10^{-5}$ & $3.2 \times 10^{-4}$ & 0.24 & {$[1,5]$} & 1024 \\
\hline
\end{tabular}

One of these parameters is the Froude number,

$$
\mathrm{Fr}=\frac{U_{0}}{\sqrt{g h_{0}}}
$$

which measures the ratio of inertia to gravity acceleration in the momentum equation, and where $U_{0}$ is the rms velocity.

Another dimensionless parameter is the nonlinear number $N_{l}$. In order to be in the regime of weak turbulence, nonlinearities should be small. The effect of nonlinearities can be measured by how large perturbations in $h$ are compared to $h_{0}$, so we define $N_{l}$ as

$$
N_{l}=\frac{h_{\mathrm{rms}}-h_{0}}{h_{0}},
$$

where $h_{\mathrm{rms}}$ is the rms value of $h$.

The two remaining dimensionless numbers are the Reynolds number,

$$
\operatorname{Re}=\frac{U_{0} L_{f}}{v}
$$

where $L_{f}$ is the forcing scale (defined as $2 \pi / k_{f_{0}}$ ), and what we will call the dispersivity $D_{s}$, defined as

$$
D_{s}=h_{0} k_{\max }=\frac{2 \pi h_{0}}{\lambda_{\min }}=\frac{N h_{0}}{6 \pi},
$$

following Eq. (30). This last number, only relevant for the Boussinesq model, measures how strong the dispersion is at the smallest scales, and for sufficiently small $D_{s}$ we can expect the solutions of the Boussinesq model to converge to the solutions of the shallow water model. In fact, it is easy to show from the weak turbulence spectrum in Eq. (25) that when the maximum resolved wave number $k_{\max }$ is associated with the dissipation wave number $k_{\eta}$, then

$$
\operatorname{Re} \sim \frac{U_{0} L_{f}}{h_{0} \epsilon^{1 / 3}} D_{s}^{5 / 3}
$$

Decreasing $D_{s}$ below the value given by this relation should result in negligible dispersion at all resolved wave numbers. Note that the level of dispersion in a given Boussinesq run depends on the wave number, and $D_{s}$ actually quantifies the strongest possible dispersion at the smallest scales in the flow.

By qualitatively assessing each run, we can classify them into three sets, $A, B$, and $C$. In Tables I, II, and III the different dimensionless parameters, along with a few other useful quantities, are given for each simulation in each set, respectively. How and why these three sets differ from each other will be made clear in the following sections, when we discuss the actual results. But, for the moment, it is fruitful to analyze the behavior of the values of $\operatorname{Re}$ and $D_{s}$ in each set, so as to keep them in mind for later on.

The values of $\operatorname{Re}$ and $D_{s}$ for all the Boussinessq runs are shown in Fig. 3. As a reference, Fig. 3 also shows the curve given by Eq. (35) with $U_{0} L_{f} /\left(h_{0} \epsilon^{1 / 3}\right)$ estimated from the values from the simulations in set $A$. Points below that curve are expected to have non-negligible dispersion. Runs in set $A$ have relatively small $\operatorname{Re}(\lesssim 1000)$, and $D_{s}$ varying between $\approx 0.02$ and $\approx 0.05$. In other words, dispersion effects in runs in set $A$ are important. Runs in set $B$ have smaller values of $D_{s}$ (except for one run with $D_{s} \approx 0.27$, all other runs have $D_{s}<0.2$ ), and Re varying between $\approx 100$ and $\approx 7000$. These runs have small or negligible dispersion, and note all the SW runs we performed belong to this set. The runs in set $C$ are intermediate between these two regimes.

TABLE III. Dimensionless numbers and parameters for runs in set $C$. Labels are as in Table I. The Boussinesq model was solved in all runs.

\begin{tabular}{lcccccrrr}
\hline \hline Simulation & Re & Fr & $D_{s}$ & $N_{l}$ & $h_{0} / L_{0}$ & $f_{0} / U_{0}$ & {$\left[k_{f_{1}}, k_{f_{2}}\right]$} & $N$ \\
\hline$C 01$ & 1400 & 0.0067 & 0.27 & $1.9 \times 10^{-5}$ & $8.0 \times 10^{-4}$ & 0.56 & {$[3,8]$} \\
$C 02$ & 1400 & 0.0073 & 0.24 & $2.4 \times 10^{-5}$ & $7.0 \times 10^{-4}$ & 0.55 & {$[3,8]$} \\
$C 03$ & 1900 & 0.0092 & 0.31 & $4.1 \times 10^{-5}$ & $4.6 \times 10^{-4}$ & 0.54 & {$[3,8]$} \\
$C 04$ & 1400 & 0.0057 & 0.43 & $1.6 \times 10^{-5}$ & $6.4 \times 10^{-4}$ & 0.74 & {$[3,8]$} & 2048 \\
$C 05$ & 470 & 0.0067 & 0.54 & $2.2 \times 10^{-5}$ & $8.0 \times 10^{-4}$ & 1.1 & {$[3,8]$} & 2048 \\
\hline \hline
\end{tabular}




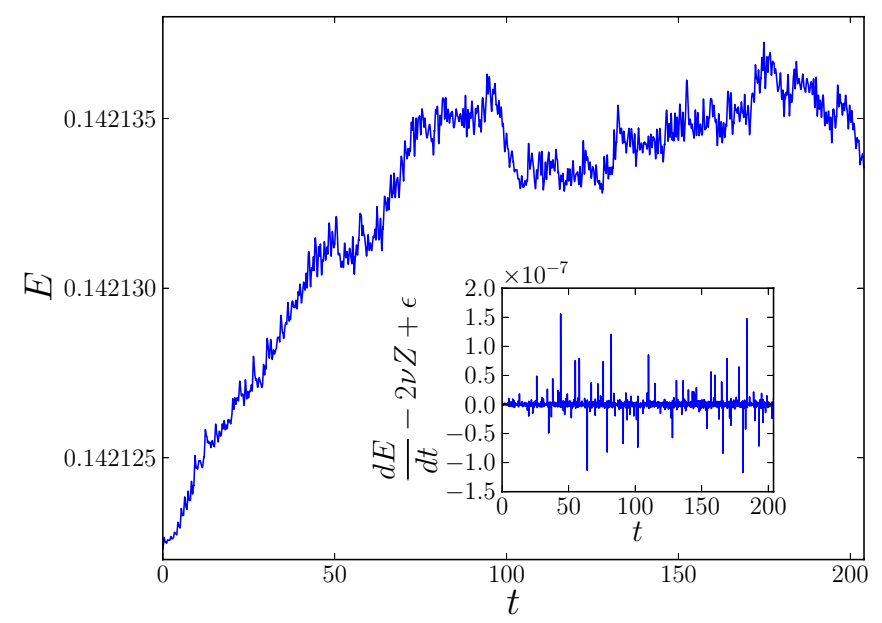

FIG. 2. (Color online) Total energy as a function of time for simulation $A 06$ (see Table I). As the fluid starts from rest and the forcing is applied, energy increases until it reaches a turbulent steady state (note energy at $t=0$ is different from zero, as the flow potential energy is never zero). All the analysis of the simulations was performed after the simulations reached the turbulent steady state. (Inset) Energy balance as a function of time [see Eq. (28)]. Note the balance is satisfied up to the seventh decimal place.

Finally, although the mechanical forcing we use introduces no vorticity in the horizontal velocity field, some vorticity is spontaneously generated as the flow evolves. This is probably also the case in experiments. In order to quantify the presence of vortical structures, we calculated the ratio of vorticity to

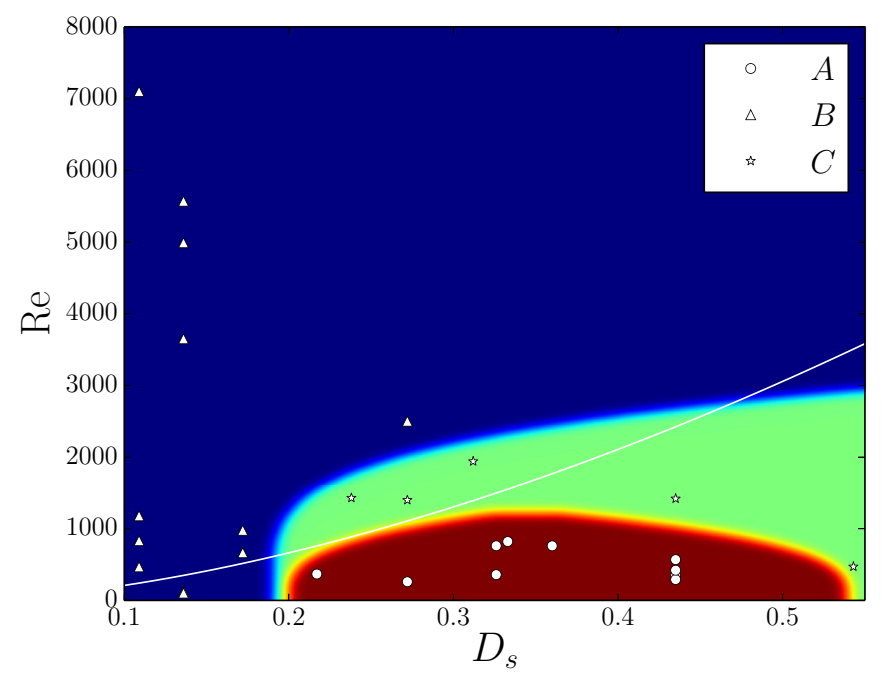

FIG. 3. (Color online) Values of the Reynolds number Re and dispersivity $D_{s}$ for all the Boussinesq runs, separated into three sets, $A$ (circles in the gray/red region), $B$ (triangles in the dark/blue region), and $C$ (stars in the light/green region), according to their different spectral behavior as discussed in Sec. IV B. The boundaries separating the three regions are arbitrary. The solid white curve corresponds to $\operatorname{Re} \sim D_{s}^{5 / 3}$; points below that curve are expected to have nonnegligible dispersion [see Eq. (35)]. Note that runs in set $A$ have relatively small Re but larger dispersion, while runs in set $B$ have either small or negligible dispersion.

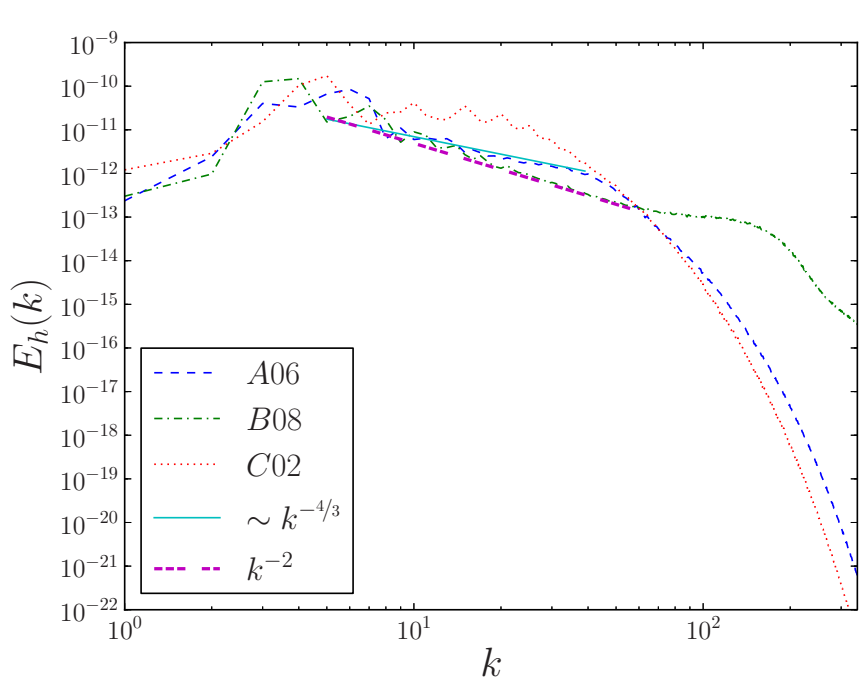

FIG. 4. (Color online) Power spectrum of $h$ (proportional to the spectrum of the potential energy) for runs $A 06$ (BQ model, $2048^{2}$ grid points, $\mathrm{Re}=360$, and $D_{s}=0.33$ ), $B 08$ (BQ model, $2048^{2}$ grid points, $\operatorname{Re}=980$, and $D_{s}=0.17$ ), and $C 02$ (BQ model, $1024^{2}$ grid points, $\operatorname{Re}=1430$, and $\left.D_{s}=0.24\right)$. Two power laws, $\sim k^{-4 / 3}$ and $\sim k^{-2}$, are shown as references.

divergence in the horizontal velocity field,

$$
\frac{\langle|\nabla \times \mathbf{u}|\rangle}{\langle|\nabla \cdot \mathbf{u}|\rangle}
$$

which turns out to be $\approx 0.1$ for all simulations. As a result, although the flow is not perfectly irrotational, the amplitude of vortical modes is small compared with the amplitude of modes associated with the waves.

\section{B. Energy spectra}

The power spectrum of $h$ (proportional to the spectrum of the potential energy) as a function of the wave number is shown in Fig. 4 for runs $A 06, B 08$, and $C 02$. Figure 5 shows a closeup

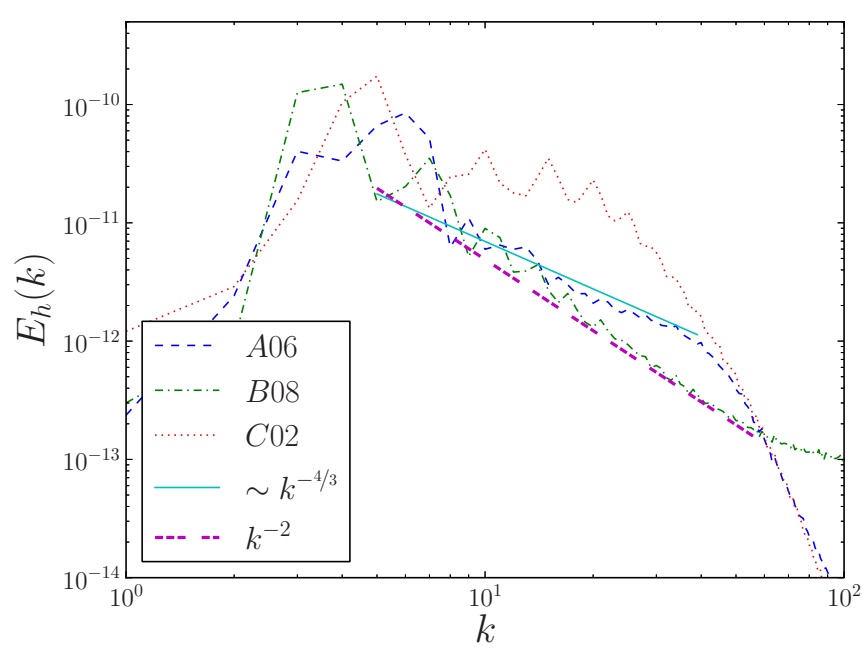

FIG. 5. (Color online) Detail of the three spectra in Fig. 4 for a subset of wave numbers to show the inertial range of the runs. Note the scaling of runs $A 06$ and $B 08$. 
of the same spectrum in the inertial range. It is clearly seen that runs in each set show a different behavior. On the one hand, the run belonging to group $A$ has an inertial range compatible with $\sim k^{-4 / 3}$ scaling, which is the spectra predicted by weak turbulence. On the other hand, the run in set $B$ displays an inertial range compatible with $\sim k^{-2}$ dependency. While this spectrum is not predicted by weak turbulence, it was observed before in experiments and observations [36]. The run in group $C$ shows a shallower spectrum with no clear inertial range. We think of runs in this set as transitional between the other two.

The other runs in sets $A, B$, and $C$ show similar power spectra for $h$. To show this, we present the compensated spectra for the simulations in sets $A$ and $B$ in Figs. 6 and 7, respectively (simulations from set $C$ do not have a clearly defined inertial range and are therefore not shown). The simulations from set $A$ are compensated by $h_{0}^{2 / 3} \epsilon^{2 / 3} k^{-4 / 3}$ (which is the weak turbulence spectrum, using the height of the fluid column at rest $h_{0}$, and the energy injection rate $\epsilon$ as prefactors), while the ones in set $B$ are compensated by $g h_{0} k^{-2}$ (more details on the choice of the prefactor are given below). These figures indicate that, within statistical uncertainties, all spectra in each set collapse to the same power laws, and that the simulations are well converged from the point of view of spatial resolution. Furthermore, we verified that the energy flux is approximately constant in the scales corresponding to the inertial range of each simulation. Within the limitations of spatial resolution and the drop in the flux for large wave numbers caused by viscous dissipation, an incipient inertial range can be identified in the flux of each simulation. Figure 8 shows the instantaneous energy flux (normalized by the energy injection rate $\bar{\epsilon}$ averaged over time) as a function of $k$ for several simulations in sets $A$ and $B$. The energy flux $\Pi(k)$ was calculated from the energy balance equation in Fourier space, as is usually done for turbulent flows. Figure 8 also shows the normalized energy dissipation rate as a function of time (equivalent to the normalized energy flux as a function of time)

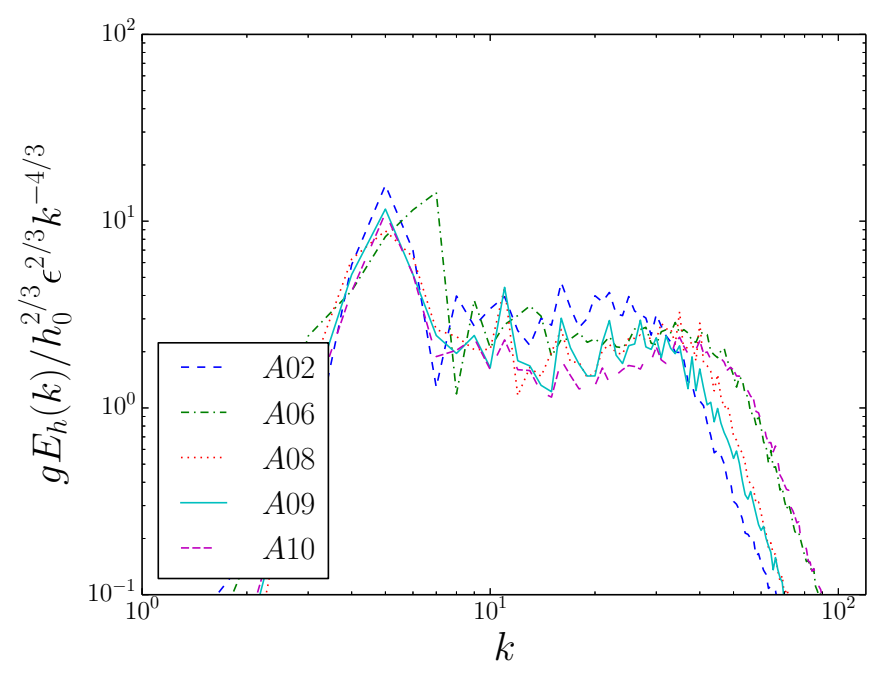

FIG. 6. (Color online) Compensated spectrum of potential energy for several simulations in set $A$. The spectra are compensated by $h_{0}^{2 / 3} \epsilon^{2 / 3} k^{-4 / 3}$. The average slope for all the runs is $-1.34 \pm 0.12$.

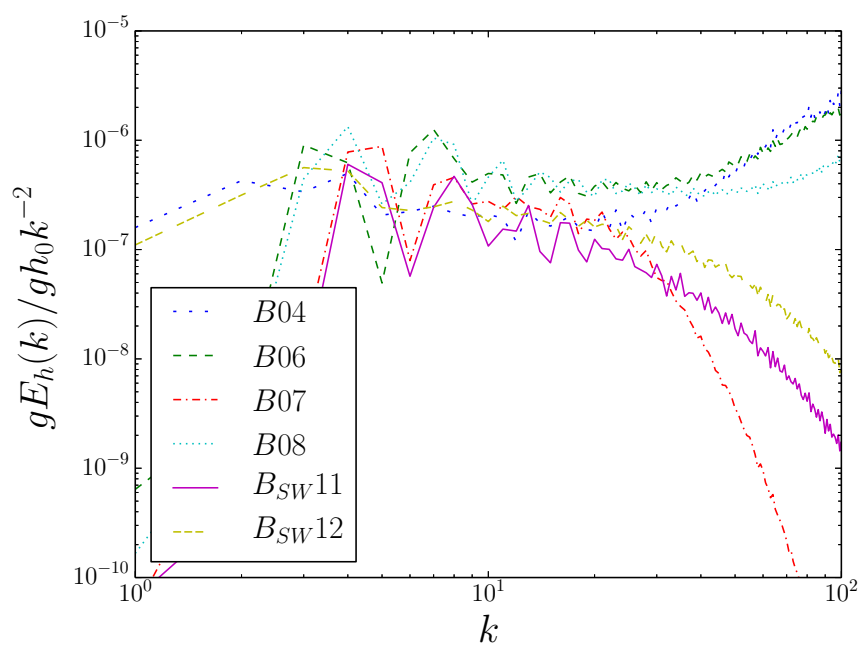

FIG. 7. (Color online) Compensated spectrum of potential energy for several simulations in set $B$. The spectra are compensated by $g h_{0} k^{-2}$. The average slope for all the runs is $-2.18 \pm 0.29$.

for the same runs, to show that this quantity fluctuates around a mean value in the turbulent steady state.

The kinetic energy spectrum is similar to the power spectrum of $h$, and in approximate equipartition with the potential energy spectrum once the system reaches a turbulent steady state. It is interesting to analyze this in light of the values of the dimensionless parameters in the runs as shown in Fig. 3. As was explained in the previous section, set $A$ corresponds to runs with lower Reynolds number and larger dispersivity $\left(\operatorname{Re} \lesssim 1000\right.$, and $D_{s}$ varying between $\approx 0.02$ and $\approx 0.05$ ). As a result, these runs can be expected to display weak turbulence behavior as described in Sec. II E, because the nonlinearities are not so large as to break the weak turbulence hypothesis [34], and the dispersion is not so low as to render the higher order terms of Eq. (14) negligible [in which case four-wave interactions would no longer be dominant, and the hypothesis used to derive Eq. (24) would not be satisfied]. In contrast, runs in set $B$ have larger Re and lower $D_{s}$ (except for one run with $D_{s} \approx 0.27$, all other runs have $D_{s}<0.2$, and $\operatorname{Re}$ varying between $\approx 100$ and $\approx 7000$ ). In this case dispersion is smaller or negligible, while nonlinearities can be expected to be larger, two conditions that render the derivation resulting in Eq. (25) invalid.

Moreover, the $\sim g h_{0} k^{-2}$ spectra observed in the simulations in set $B$ include those runs that solve the SW model. Therefore, these spectra cannot be explained by weak turbulence, as SW simulations have no dispersion and the arguments in Sec. II E do not apply. In addition, note that in the nondispersive limit, for constant and fixed $h$, the SW equations can be reduced to the two-dimensional Burgers equations, which amplify negative field gradients by strong nonlinearities resulting in sharp fronts in the velocity. Such a field would actually have a spectrum $\sim k^{-2}$ (note this is also the behavior expected for two-dimensional nondispersive acoustic turbulence [50], that also develops sharp fronts). The spectrum can be obtained from dimensional analysis and the scaling that results for the energy is equivalent to Phillips' spectrum [27] but in two dimensions. In the presence of strong nonlinearities, we can assume that 

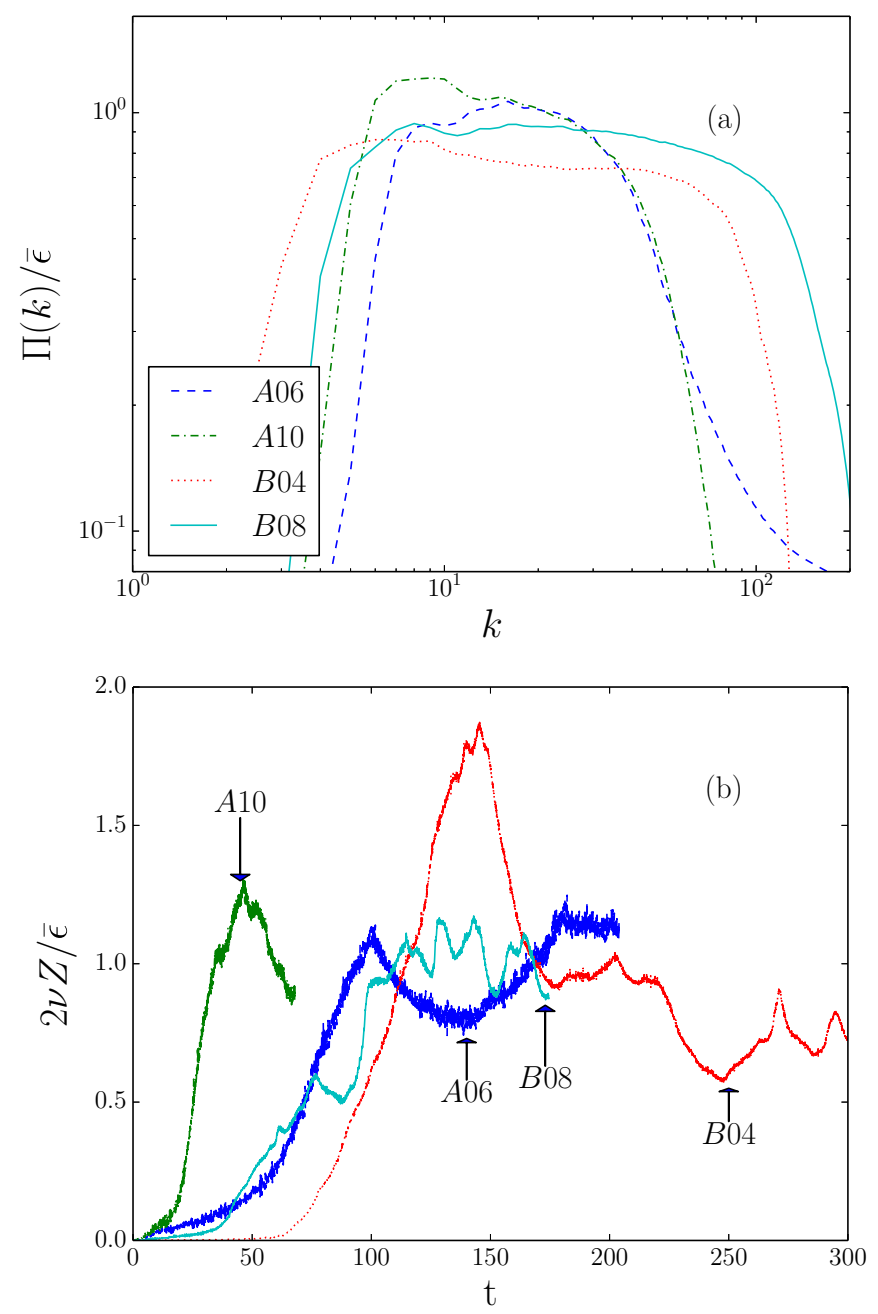

FIG. 8. (Color online) (a) Energy flux (normalized by the mean energy injection rate) as a function of $k$. For each simulation, a range of wave numbers can be identified for which $\Pi(k)$ remains approximately constant, and this range is in reasonably good agreement with the inertial ranges identified in Figs. 6 and 7. (b) Energy dissipation rate (normalized by the averaged in time energy injection rate) as a function of time for the same simulations.

the nonlinear and gravity terms are of the same order,

$$
\mathbf{u} \cdot \nabla \mathbf{u} \sim g \nabla h .
$$

It is also reasonable to assume that the kinetic and potential energies will be of the same order (i.e., in equipartition) in the turbulent steady state. This implies that $g$ is the only dimensional constant the spectra can depend on. This is precisely how Phillips derived his spectrum.

With these assumptions in mind, it is easy to obtain the observed spectra. The energy spectrum has units of energy in the fluid column per unit surface per wave number, $E(k) \sim$ $h_{0} u^{2} / k$, and assuming $E(k) \sim g h_{0} k^{-\alpha}$, from dimensional analysis the only possible solution is

$$
E(k) \sim g h_{0} k^{-2} .
$$

The independence of the spectrum on the energy injection rate suggests that the energy transfer between the different scales must take place by a mechanism such as wave breaking in the case of Phillips' spectrum, which occurs when the slope of the surface is larger than a critical value, or by nonlinear wave steepening in our case (which is finally regularized by the viscosity). Such a mechanism is independent of the power injected by external forces. Of course, this can only hold in a region of parameter space because in the presence of weak forcing and dispersion, the solution in Eq. (25) is expected instead.

In summary, based on the numerical results, the simulations with weaker forcing and higher dispersion develop a spectrum compatible with the predictions from weak turbulence theory, while the runs with stronger forcing or with less (or no) dispersion are compatible with dimensional analysis based on strong turbulence arguments.

\section{Comparison between SW and BQ models}

All simulations of the SW model belong to set $B$, as that is the set of runs that has negligible or no dispersion. All other sets have moderate dispersion, and as a result the flow dynamics cannot be captured by the SW model. Note that runs in set $B$ are also the runs with an inertial range compatible with $\sim k^{-2}$ scaling. However, the $\mathrm{BQ}$ and $\mathrm{SW}$ runs in set $B$ are not identical. In this subsection we discuss the differences between these runs.

As an example of two runs with and without dispersive effects, the power spectra of $h$ for runs $B 04$ and $B_{\mathrm{SW}} 12$ are shown in Fig. 9. Both simulations have the same parameters, except for the viscosity which is larger in the simulation using the SW model. At small wave numbers, where dispersion is negligible, the spectra of the $\mathrm{BQ}$ and $\mathrm{SW}$ models coincides. For wave numbers larger than $\approx 30$, dispersion in the $\mathrm{BQ}$ model becomes important and a bump (an accumulation of energy at small scales) develops. This accumulation in the BQ model results in an increased dissipation [as dissipation is proportional to $k^{2} E(k)$ ], thus allowing us to simulate the

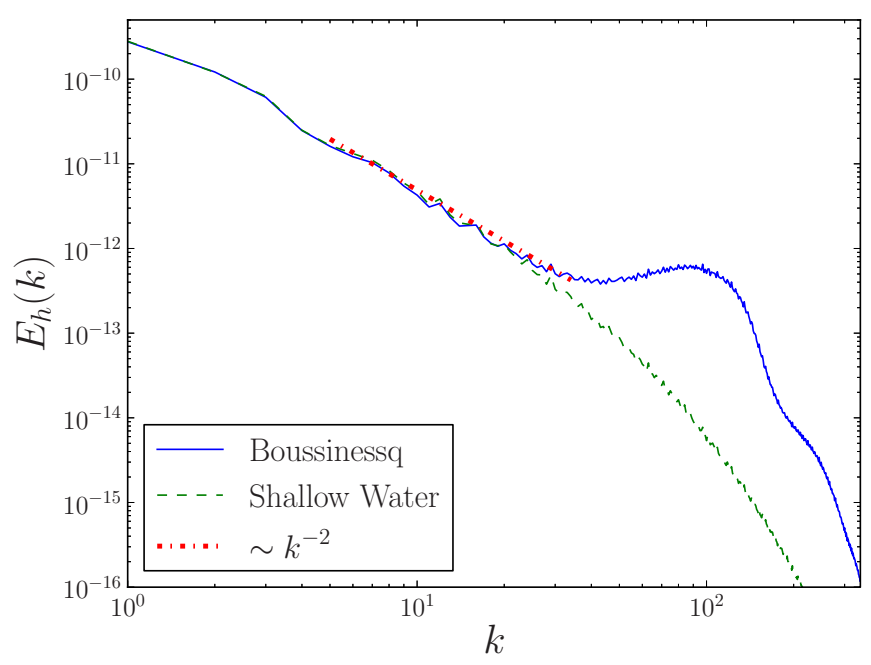

FIG. 9. (Color online) Power spectrum of $h$ for simulations $B 04$ and $B_{\mathrm{SW}} 12$. The former corresponds to a numerical solution of the BQ model, while the latter to a solution of the SW model. A $\sim k^{-2}$ power law is indicated as a reference. 


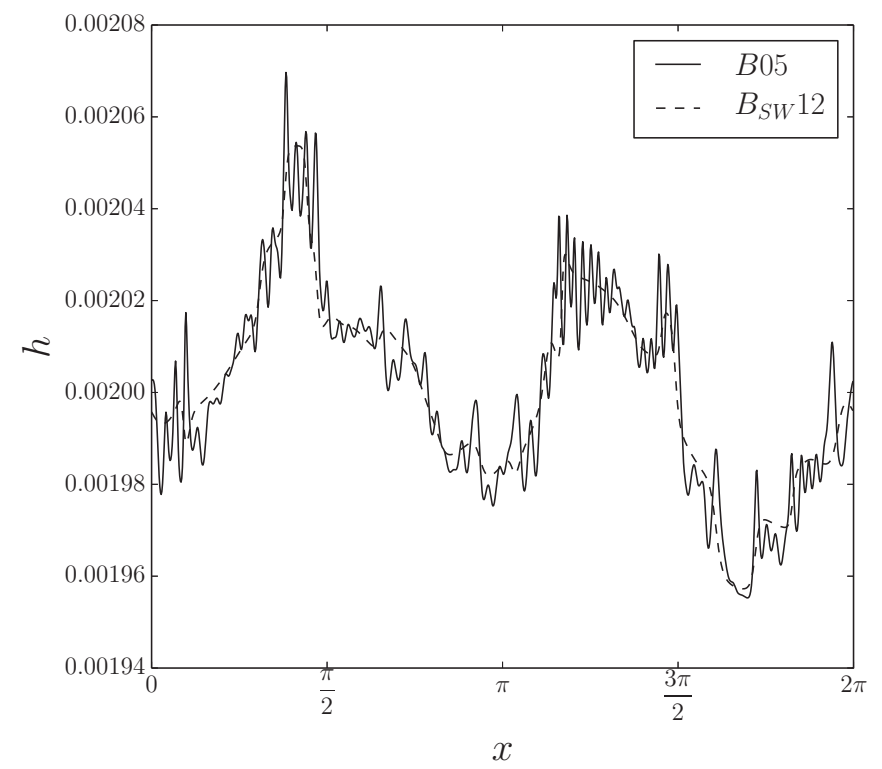

FIG. 10. One-dimensional cut of the height $h$ in the turbulent steady state of runs $B 04$ and $B_{\mathrm{SW}} 12$, at the same time. The former run corresponds to a numerical solution of the BQ model, while the latter to a solution of the SW model. While the long length scales show the same behavior in both runs, note the BQ model has larger fluctuations at short length scales. Both runs were computed with a linear resolution of $N=1024$ grid points, and the fast fluctuations are well resolved.

system with smaller viscosity. This difference at large wave numbers is the most distinct feature in the two spectra in Fig. 9.

As a result of the extra power at larger wave numbers, dispersion in the BQ model results in more prominent smallscale features, and in rapidly varying waves. As an example, Fig. 10 shows a transversal cut in the elevation field for runs $B 04$ and $B_{\mathrm{SW}} 12$. The cuts are taken at the same place and at the same time in both runs. Even though both simulations have the same behavior at large scales, at short length scales the BQ model presents fast fluctuations. These fluctuations are well resolved (the cut corresponds to 1024 grid points), and there is no indication that resolution is insufficient to resolve the sharp gradients. In the BQ model, while the large scales may correspond to a shallow flow, as long as there is enough scale separation, there will always be a wave number where the finite depth effects can be seen. Thus, the Boussinesq equations provide an interesting model to study weakly dispersive waves.

Regarding the accumulation of energy that leads to a flatter spectrum for high wave numbers in some of the BQ simulations (for several runs in set $B$ as can be seen in Fig. 7, but especially in the runs in set $C$ ), such an accumulation has been observed before in turbulent flows. As mentioned above, we verified that this accumulation is not the result of insufficient resolution (e.g., by comparing the runs with different grid points $N$ ). The accumulation of energy in the spectrum near the dissipative range is often termed "bottleneck," and bottlenecks can have dissipative [55] or dispersive [49,56] origins. In the former case, the accumulation results from the viscous damping of the triads at small scales, resulting in a decrease of the energy flux. Such a viscous bottleneck should be visible also in the nondispersive simulations, and its absence in those runs indicates a dispersive origin. In the latter case, the bottleneck arises from the increasingly harder to satisfy resonant condition for the wave frequencies, as the waves become faster at smaller scales. Models with a field filtered by the Helmholtz operator [as is the case for the BQ model; see Eq. (15)] tend to develop a bottleneck (see [49] for a detailed description of its origin). A qualitative way to explain the tendency towards a flatter spectrum in the BQ model can be obtained by assuming that dispersion is strong enough for the dispersive term to be balanced with the buoyancy and with the nonlinear terms in the BQ equations (i.e., all terms are of the same order). Then the energy spectra can depend only on both $g$ and $h_{0}$, and a possible solution is $E(k) \sim g h_{0}^{2}$. A detailed study of the origin of this bottleneck is left for future work.

At this point it is worth pointing out that when $D_{s} \approx 1$ and dispersion becomes too strong, the Boussinesq approximation breaks down as more terms in the Taylor expansion in Eq. (11) should be preserved. As a result, the Boussinesq approximation is useful as long as $D_{s}<1$ at the smallest excited scales in the system. On the other hand, from Fig. 3, if $D_{s} \lesssim 0.15$ the behavior of the system in the inertial range is that of a shallow water flow for all Reynolds numbers studied.

\section{Time-resolved spectra and nonlinear dispersion relations}

Wave-number spectra, as the spectra discussed so far, give information on how energy is distributed in spatial scales, but do not provide a quantitative estimate of how much energy in the system is associated with wave motions. A frequency spectrum $E(\omega)$ is often obtained from the wave-number spectrum $E(k)$ using the dispersion relation (or vice versa). However, in systems that can sustain both wave and vortical motions there is no clear justification to use the dispersion relation to go from one spectrum to the other.

A quantification of the amount of energy in waves, and on whether nonlinear effects change the dispersion relation of the system from the linear one, can be directly obtained from the frequency and wave-number spectrum $E(k, \omega)$ without any assumption. The spectrum $E(k, \omega)$ can be computed by storing the Fourier coefficients of the height $\hat{h}(\mathbf{k}, t)$ as a function of time (as well as the Fourier coefficients of the velocity field), then computing the Fourier transform in time, and finally computing the isotropic power spectrum by averaging in the $\left(k_{x}, k_{y}\right)$ plane. To this end, several large-scale wave periods and turnover times must be stored (to resolve the slowest frequencies in the system), with sufficient time resolution $\Delta t$ to resolve the fastest frequencies. In the analysis we show below, time series spanning at least three periods of the slowest waves were used, and with time resolution $\Delta t \approx 3 \times 10^{-4}$.

Figures 11 and 12 show the power spectrum of the flow height $E_{h}(k, \omega)$ for simulations $B 04$ and $A 02$, respectively. The linear dispersion relations for shallow water flows [Eq. (4)] and for Boussinesq flows [Eq. (14)] are also shown as references, using the parameters from each run. Note both runs present an energy accumulation near the dispersion relation. This indicates most of the energy is in the waves, and remains there as time evolves. As we are not solving the equations for a potential flow, and the system can develop vortical 

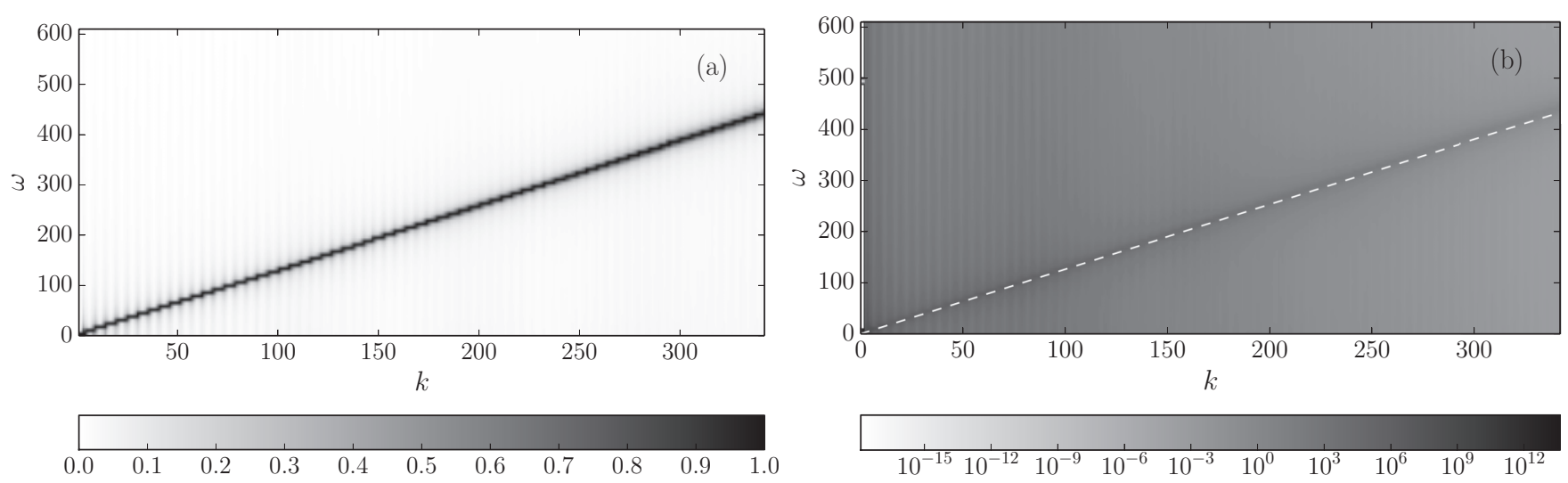

FIG. 11. Power spectrum $E_{h}(k, \omega)$ for simulation $B 04$. The darker regions correspond to larger power density, while the lighter regions correspond to smaller power density. (a) Normalized power spectrum $E_{h}(k, \omega) / E_{h}(k)$. (b) Non-normalized power spectrum. The white dashed line appearing in the bottom panel indicates the linear dispersion relation from Eq. (14). Note that as in this run dispersion is negligible, the dispersion relation is almost that given by Eq. (4), and nondispersive.

motions, this tells us that the nonlinear energy transfer is mostly done between waves, and that the energy injected at large scales in wave motions is mostly transferred towards wave motions at smaller scales and faster frequencies. This is needed for weak turbulence to hold, but is also observed in run $B 04$ that has a spectrum compatible with strong turbulence phenomenological arguments. There is also a turbulent broadening of the dispersion relation, also visible in cross sections of the spectrum at different wave numbers in Fig. 13. From this broadening, the characteristic time of nonlinear wave interactions can be obtained, as was done in [57].

Some of the most important results in this paper are associated with these two figures. First, note that in run $B 04$ most of the energy is concentrated near a dispersion relation that, as dispersion is negligible, corresponds in practice to the nondispersive shallow-water case [Eq. (4)]. All runs in set $B$ have the same spectral behavior in $E_{h}(k, \omega)$, and confirm that the $\sim k^{-2}$ spectrum is observed when dispersion is negligible or absent (i.e., when the flow is sufficiently shallow). Second, note that the spectrum $E_{h}(k, \omega)$ in run $A 02$ presents clear signs of dispersive effects (i.e., most of the energy for large enough $k$ is concentrated over a curve that deviates from a linear relation between $k$ and $\omega$ ), and this run displays a scaling in $E_{h}(k)$ compatible with the weak turbulence prediction $\sim k^{-4 / 3}$. This behavior was observed in the other runs in set $A$.

However, $E_{h}(k, \omega)$ for runs in set $A$ presents yet another interesting feature. As expected, for small $k$ the dispersion is negligible and the energy is concentrated over a straight line in $(k, \omega)$ space. At large $k$, as already mentioned, the effective dispersion relation is compatible with that of the linearized Boussinesq equations. But at intermediate wave numbers two branches of the dispersion relation can be observed, one that is compatible with nondispersive waves and another compatible with dispersive waves. When both branches are present, their amplitudes are of the same order, as can be seen in Fig. 13.

At first sight, the existence of these two branches could be attributed to bound waves. Bound waves are small amplitude waves which are bounded to a parent wave of larger amplitude. The waves are bounded in the sense that they follow the parent wave, i.e., they travel with the same phase velocity as the parent, and thus they follow an anomalous dispersion relation (see, e.g., a discussion of bound waves in the context
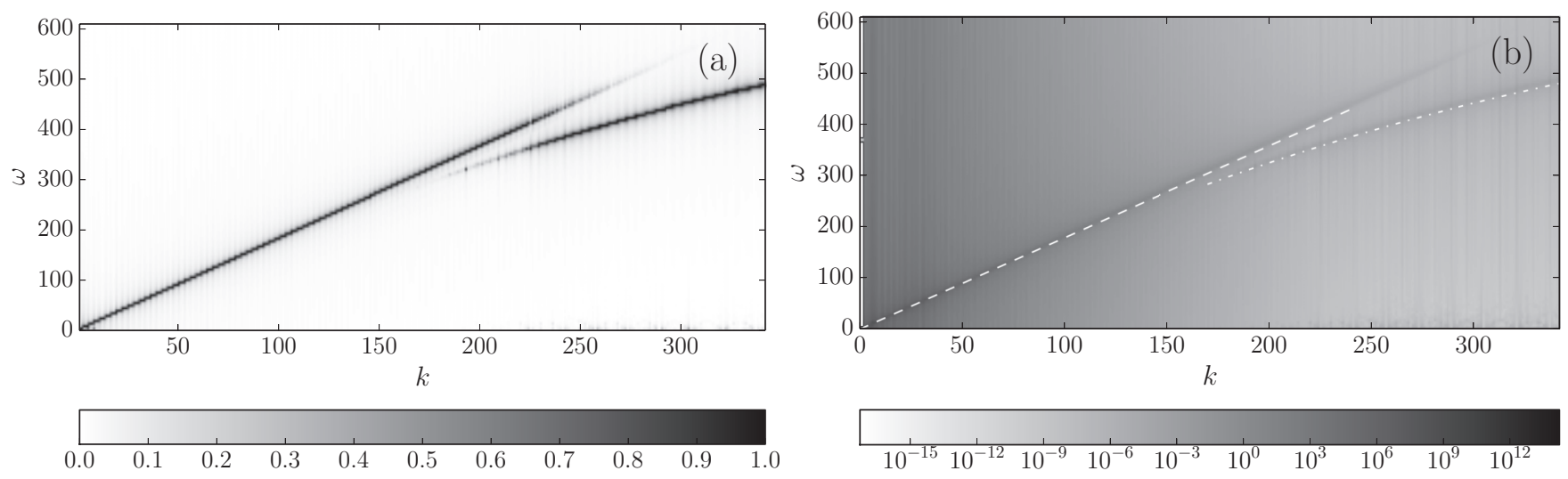

FIG. 12. Power spectrum $E_{h}(k, \omega)$ for simulation $A 02$. The darker regions correspond to larger power density, while the lighter regions correspond to smaller power density. (a) Normalized power spectrum. (b) Non-normalized power spectrum. The white dashed line appearing in the bottom panel indicates the (nondispersive) linear dispersion relation from Eq. (4), and the white dash-dotted line indicates the BQ dispersion relation from Eq. (14). 


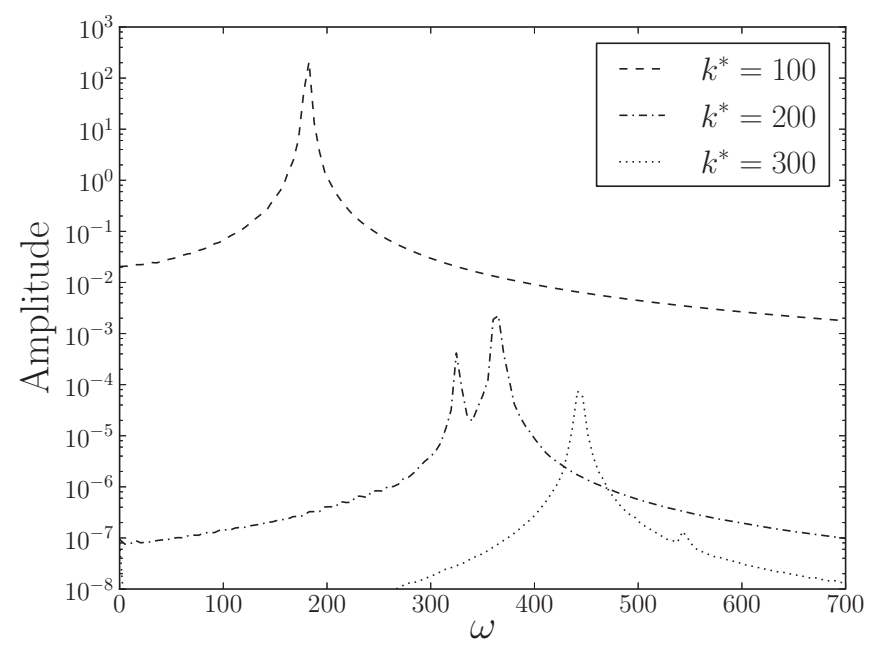

FIG. 13. Cross sections of $E_{h}(k, \omega)$ at different (and fixed) values of $k=k^{*}$ for run $A 02$. Note the peaks and surrounding wave numbers have most of the power. Note also the two peaks for $k=200$, one corresponding to the shallow-water dispersion relation, and the other to the Boussinesq dispersion relation.

of gravito-capillary waves in [58,59]). The condition that they have the same phase velocity as the parent wave implies that they must follow a modified dispersion relation which verifies $\Omega(k)=\omega\left(k_{0}\right) k / k_{0}$, where $k_{0}$ is the wave number of the parent wave. Bound waves result in multiple branches in the $E(k, \omega)$ spectrum (and in multiple peaks in the frequency spectrum). Indeed, it is easy to show that for $k=N k_{0}, N=2,3,4, \ldots$, these multiple branches satisfy

$$
\Omega_{N}\left(N k_{0}\right)=N \omega\left(k_{0}\right)
$$

(see, e.g., the discussion in [59]). Extending the analysis in [59] to our case, bound waves in the BQ model should satisfy the following dispersion relation,

$$
\Omega_{N}(N k)=\frac{c_{0} k}{\sqrt{1+\frac{h_{0}^{2} k^{2}}{3 N^{2}}}},
$$

which verifies Eq. (39). However, the second branch in Fig. 12 cannot be described by this dispersion relation for any value of $N$ up to 4 , and thus they are not bound waves in the sense often used in oceanography.

Another explanation for the existence of these two branches can be given by keeping in mind that at intermediate wave numbers slight variations in the fluid depth may trigger a transition in the waves from dispersive to nondispersive (as the level of dispersion depends on the product of the wave number with the surface height). Indeed, in the turbulent flow there are waves with short wavelengths which ride over long ones, that have a larger amplitude. For sufficient scale separation, the fast waves see an effective depth that can be larger or smaller than $h_{0}$ depending on whether the wave is on a crest or a valley of the slow wave, generating in one case dispersive waves, and in the other nondispersive waves.

We can estimate the variation in the effective dispersion at a given wave number $k$. In simulation $A 02, h_{0}=4 \times 10^{-3}$ and the longer waves have an amplitude $\delta \approx 4 \times 10^{-5}$ (as can be estimated, e.g., from the maximum value of the power spectrum of $h)$. From the system dispersion relation,

$$
\omega^{2}=c_{0}^{2} k^{2}\left(1-\frac{1}{3} h_{0}^{2} k^{2}\right)
$$

dispersion is controlled by the amplitude of the $h_{0}^{2} k^{2} / 3$ term. Assuming that fast waves experience an effective depth $h_{0} \pm$ $\delta$ (where the sign depends on whether they are on a valley or a crest), the variation in the dispersion is proportional to the difference between $\left(h_{0}-\delta\right)^{2}$ and $\left(h_{0}+\delta\right)^{2}$. So, for this simulation, the variation is around $4 \%$, and when multiplied by $k^{2}$, it is sufficient to explain the two branches in $E_{h}(k, \omega)$ for $k$ between $\approx 150$ and 250 .

\section{E. Time frequency energy spectra}

From the spectra in Figs. 11 and 12 the frequency spectrum $E_{h}(\omega)$ can be easily obtained, simply by summing over all wave numbers,

$$
E_{h}(\omega)=\sum_{k} E_{h}(k, \omega) .
$$

As already mentioned, in experiments and simulations $E_{h}(\omega)$ is sometimes estimated instead from $E_{h}(k)$ by using the dispersion relation in the form $k=k(\omega)$. Figure 14 shows the power spectrum of $h$ as a function of $\omega$ for simulations $A 02$ and $B 04$. In both cases, the spectrum was calculated explicitly using Eq. (42), and also estimated using the dispersion relation. For each run, the two spectra show a very good agreement, which can be expected as most of the energy is in the waves. The behavior of the inertial range in each run is also in good agreement with the one found previously for $E_{h}(k)$ in Sec. IV B.

\section{F. Probability density functions}

We calculated the probability density function (PDF) of the free surface height for different simulations. Figure 15 shows the PDF of $h / \sigma$ for run A06, where $\sigma$ is the standard deviation of the surface height. The probability distribution is asymmetric, with a larger probability of measuring large values of $h$ than of small values. The shape can be adjusted by two distributions: We consider a skewed normal distribution [60],

$$
f(x)=\frac{2}{\kappa} \phi\left(\frac{x-\xi}{\kappa}\right) \Phi\left(\alpha \frac{x-\xi}{\kappa}\right),
$$

where $\kappa$ is the so-called scale parameter (associated with the variance of the distribution), $\xi$ is the location parameter (associated with the mean value), $\alpha$ is the shape parameter (associated with the skewness), and

$$
\begin{gathered}
\phi(x)=\frac{1}{\sqrt{2 \pi}} e^{-x^{2} / 2}, \\
\Phi(x)=\int_{-\infty}^{x} \phi(t) \mathrm{d} t=\frac{1}{2}\left[1+\operatorname{erf}\left(\frac{x}{\sqrt{2}}\right)\right] .
\end{gathered}
$$

We also consider a Tayfun distribution,

$$
p(x)=\int_{0}^{\infty} \frac{e^{-\left[y^{2}+(1-c)^{2}\right] /\left(2 s^{2}\right)}}{\pi s c} d y,
$$

with $c=\sqrt{1+2 s x+y^{2}}$ and where $s$ is the mean steepness of the waves [61]. 

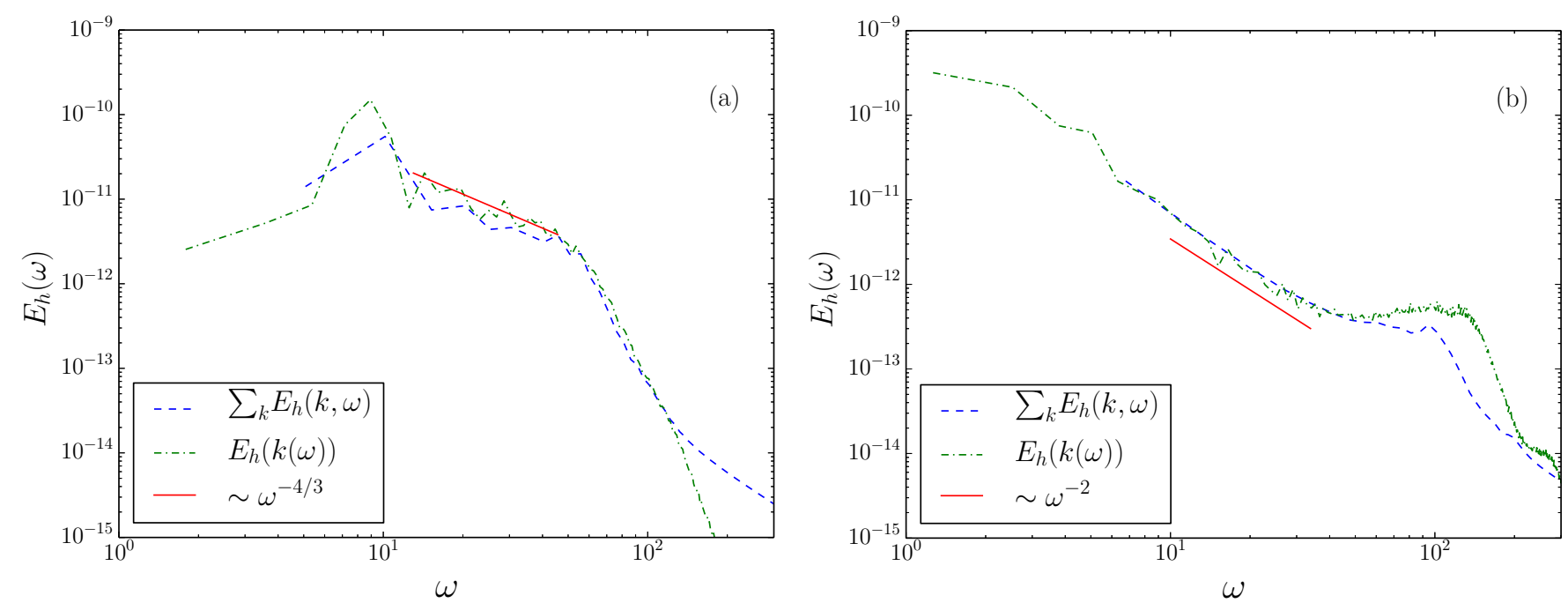

FIG. 14. (Color online) Power spectrum of $h$ as a function of the frequency for simulations (a) $A 02$ and (b) B04. In both cases, the spectrum was calculated by summing over all wave numbers in the time- and space-resolved spectrum, $\sum_{k} E_{h}(k, \omega)$, and also by using the dispersion relation given by Eq. (14) to estimate the frequency spectrum from the wave-number spectrum $E_{h}(k)$. As a reference, power laws $\sim \omega^{-4 / 3}$ and $\sim \omega^{-2}$ are shown in each case. The behavior is in good agreement with the one found for $E_{h}(k)$.

For run A06, and from a Maximum Likelihood Estimation method for the skewed normal distribution, the location parameter is $\xi \approx-1.00$, the scale parameter is $k \approx 1.43$, and the shape parameter is $\alpha \approx 1.94$. For the same run, and for the Tayfun distribution, the mean steepness of the waves is $s \approx 0.15$. This latter value is more relevant as the Tayfun distribution is often used in oceanography and in experiments of surface waves. In this context, it is interesting to point out that experiments in [37] found similar values for $s$.

This behavior (a PDF of $h$ described correctly by both a skewed normal distribution and a Tayfun distribution with

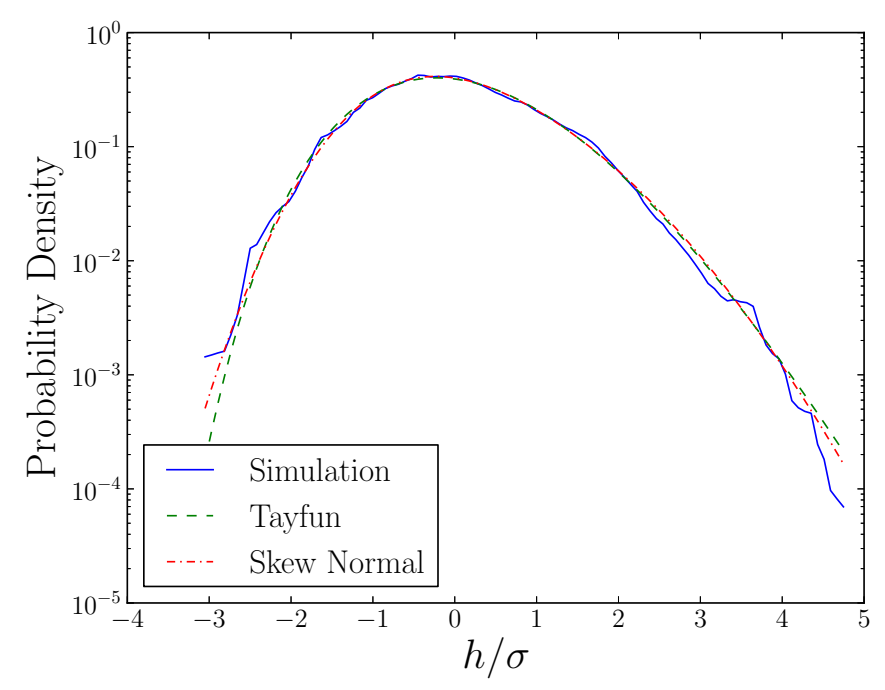

FIG. 15. (Color online) Probability density function of the values of $h$ in simulation $A 06$ (solid blue line). The dash-dotted (red) line indicates a maximum likelihood fit using a skewed normal distribution, while the dashed (green) line corresponds to a maximum likelihood fit for the Tayfun distribution. asymmetry to the left) was observed in all simulations, no matter what set they belonged to.

\section{CONCLUSIONS}

We studied wave turbulence in shallow water flows in numerical simulations using the shallow water and Boussinesq models. The equations were solved using grids up to $2048^{2}$ points, and the parameters were varied to study different regimes, including regimes with larger and smaller Reynolds number, and larger and smaller dispersion, while keeping the Froude number approximately the same. We summarize below the main conclusions following the same ordering as in the introduction:

(1) As in previous experimental and observational studies $[35,36]$, we found that the flows can be classified in different sets depending on the value of the Reynolds number (i.e., on the strength of the nonlinearities) and on the level of dispersion (associated with the fluid depth). A first set $(A)$ has smaller Reynolds numbers and stronger dispersion, a second set $(B)$ has larger Reynolds numbers and weaker or negligible dispersion, and a third set of runs seems to be transitional between the two.

(2) Runs in sets $A$ and $B$ have different power spectra of the surface height. Runs in set $A$, with stronger dispersion, present a spectrum compatible (within statistical uncertainties) with $E_{h}(k) \sim k^{-4 / 3}$. This is the spectrum predicted by weak turbulence theory for the Boussinesq equations [34]. Runs in set $B$ with negligible or zero dispersion (i.e., for a shallower flow) show a spectrum compatible within error bars with $E_{h}(k) \sim k^{-2}$. This spectrum can be obtained from phenomenological arguments coming from strong turbulence [27]. The runs in set $C$ have no discernible inertial range.

(3) The Boussinesq (dispersive) model tends to develop more power in waves with short wavelengths than the shallow 
water model. This is associated with the development of a bottleneck for large wave numbers in the energy spectrum.

(4) Inspection of the wave and frequency spectrum $E_{h}(k, \omega)$ confirms that most of the energy is in the waves in all the simulations. In runs in set $B$, most of the energy is concentrated in the vicinity the linear dispersion relation for shallow water waves, which are nondispersive. In runs in set $A$, the resulting nonlinear dispersion relation obtained from $E_{h}(k, \omega)$ has two branches: one that corresponds to nondispersive waves, and another corresponding to dispersive waves. The two branches can be explained as the result of the superposition of rapidly varying waves which ride over slowly varying waves, the latter with sufficient amplitude to change whether the former see a shallower or deeper fluid.

(5) Independently of the differences between the runs, the probability distribution functions of $h$ for the runs in all sets is asymmetric, with larger probabilities of finding larger values of $h$ than smaller values. The probability distribution functions can be approximated by both a skewed normal distribution and a Tayfun distribution [61]. In the latter case, the only parameter of the distribution, the mean steepness of the waves, has values compatible with those found in observations and experimental studies (see [37]). The obtained probability density functions also indicate limitations in the hypothesis of Gaussianity of the fields assumed in early theories of weak turbulence. However, extensions of the theory to allow for non-Gaussian distributions exist and can be found, for example, in Refs. [62-64].

All the results presented here were obtained solving numerically equations that do not assume that the flow is inviscid or irrotational, and with realistic terms for the viscous dissipation. We believe this approach can be useful to compare with experiments because in experiments vorticity can develop in the flow, and viscosity cannot be neglected.

\section{ACKNOWLEDGMENTS}

The authors would like to thank Professor Oliver Buhler and the anonymous referees for their useful comments. The authors acknowledge support from Grants No. PIP 11220090100825 , No. UBACYT 20020110200359, and Nos. PICT 2011-1529 and 2011-1626. P.D.M. and P.J.C. acknowledge support from the Carrera del Investigador Científico of CONICET, and P.C.dL. acknowledges support from CONICET.
[1] A. Iafrati, A. Babanin, and M. Onorato, Phys. Rev. Lett. 110, 184504 (2013).

[2] E. D’Asaro, C. Lee, L. Rainville, R. Harcourt, and L. Thomas, Science 332, 318 (2011).

[3] G. Ivey, K. Winters, and J. Koseff, Annu. Rev. Fluid Mech. 40, 169 (2008).

[4] K. A. Rose, E. L. Sikes, T. P. Guilderson, P. Shane, T. M. Hill, R. Zahn, and H. J. Spero, Nature (London) 466, 1093 (2010).

[5] L. Cavaleri, B. Fox-Kemper, and M. Hemer, Bull. Am. Meteorol. Soc. 93, 1651 (2012).

[6] J. Falnes, Marine Structures 20, 185 (2007).

[7] K. Hasselmann, J. Fluid Mech. 12, 481 (1962).

[8] K. Hasselmann, J. Fluid Mech. 15, 385 (1963).

[9] K. Hasselmann, J. Fluid Mech. 15, 273 (1963).

[10] V. E. Zakharov, V. S. Lvov, and G. Falkovic, Kolmogorov Spectra of Turbulence I-Wave Turbulence (Springer, Berlin, 1992).

[11] A. C. Newell and B. Rumpf, Annu. Rev. Fluid Mech. 43, 59 (2011).

[12] S. Nazarenko, Wave Turbulence, 1st ed. (Springer, Berlin, 2011).

[13] G. Düring, C. Josserand, and S. Rica, Phys. Rev. Lett. 97, 025503 (2006).

[14] S. Galtier, Phys. Rev. E 68, 015301 (2003).

[15] S. Galtier, S. V. Nazarenko, A. C. Newell, and A. Pouquet, J. Plasma Phys. 63, 447 (2000).

[16] A. A. Schekochihin, S. V. Nazarenko, and T. A. Yousef, Phys. Rev. E 85, 036406 (2012).

[17] L. Deike, M. Berhanu, and E. Falcon, Phys. Rev. E 85, 066311 (2012).

[18] C. Falcón, E. Falcon, U. Bortolozzo, and S. Fauve, Europhys. Lett. 86, 14002 (2009).

[19] G. V. Kolmakov, A. A. Levchenko, M. Y. Brazhnikov, L. P. Mezhov-Deglin, A. N. Silchenko, and P. V. E. McClintock, Phys. Rev. Lett. 93, 074501 (2004).
[20] P. Cobelli, A. Przadka, P. Petitjeans, G. Lagubeau, V. Pagneux, and A. Maurel, Phys. Rev. Lett. 107, 214503 (2011).

[21] N. Mordant, Phys. Rev. Lett. 100, 234505 (2008).

[22] A. Boudaoud, O. Cadot, B. Odille, and C. Touzé, Phys. Rev. Lett. 100, 234504 (2008).

[23] P. Cobelli, P. Petitjeans, A. Maurel, V. Pagneux, and N. Mordant, Phys. Rev. Lett. 103, 204301 (2009).

[24] S. Leerink, V. V. Bulanin, A. D. Gurchenko, E. Z. Gusakov, J. A. Heikkinen, S. J. Janhunen, S. I. Lashkul, A. B. Altukhov, L. A. Esipov, M. Y. Kantor, T. P. Kiviniemi, T. Korpilo, D. V. Kuprienko, and A. V. Petrov, Phys. Rev. Lett. 109, 165001 (2012).

[25] P. D. Mininni and A. Pouquet, Phys. Rev. Lett. 99, 254502 (2007).

[26] Q. Chen, S. Chen, G. L. Eyink, and D. Holm, J. Fluid Mech. 542, 139 (2005).

[27] O. M. Phillips, J. Fluid Mech. 4, 426 (1958).

[28] Y. Toba, Journal of the Oceanographical Society of Japan 29, 209 (1973).

[29] M. A. Donelan, J. Hamilton, and W. H. Hui, Philos. Trans. R. Soc. London A 315, 509 (1985).

[30] S. I. Badulin, A. V. Babanin, V. E. Zakharov, and D. Resio, J. Fluid Mech. 591, 339 (2007).

[31] O. M. Phillips, J. Fluid Mech. 156, 505 (1985).

[32] A. O. Korotkevich, Phys. Rev. Lett. 101, 074504 (2008).

[33] V. Zakharov, European Journal of Mechanics, B/Fluids 18, 327 (1999).

[34] M. Onorato, A. R. Osborne, P. A. E. M. Janssen, and D. Resio, J. Fluid Mech. 618, 263 (2009).

[35] J. M. K. Smith and C. L. Vincent, J. Geophys. Res. 108, 3366 (2003).

[36] J. M. Kaihatu, J. Veeramony, K. L. Edwards, and J. T. Kirby, J. Geophys. Res. 112, C06016 (2007).

[37] E. Falcon and C. Laroche, Europhys. Lett. 95, 34003 (2011). 
[38] L. D. Landau and E. M. Lifshitz, Fluid Mechanics (Elsevier/Butterworth-Heinemann, Amsterdam, 2004).

[39] J. Pedlosky, Geophysical Fluid Dynamics, 2nd ed. (SpringerVerlag, New York, 1987).

[40] G. B. Whitham, Linear and Nonlinear Waves (Wiley, New York, 1974).

[41] A. I. Dyachenko, A. O. Korotkevich, and V. E. Zakharov, Phys. Rev. Lett. 92, 134501 (2004).

[42] N. Yokoyama, J. Fluid Mech. 501, 169 (2004).

[43] F. Marche, European Journal of Mechanics, B/Fluids 26, 49 (2007).

[44] W. Choi, J. Fluid Mech. 295, 381 (1995).

[45] P. D. Mininni, D. C. Montgomery, and A. G. Pouquet, Phys. Fluids 17, 035112 (2005).

[46] P. D. Mininni, D. C. Montgomery, and A. Pouquet, Phys. Rev. E 71, 046304 (2005).

[47] C. Foias, D. D. Holm, and E. S. Titi, Physica D: Nonlinear Phenomena 152-153, 505 (2001).

[48] R. Camassa and D. D. Holm, Phys. Rev. Lett. 71, 1661 (1993).

[49] J. Pietarila Graham, D. D. Holm, P. D. Mininni, and A. Pouquet, Phys. Rev. E 76, 056310 (2007).

[50] V. S. L'vov, Y. L'vov, A. C. Newell, and V. Zakharov, Phys. Rev. E 56, 390 (1997).
[51] D. O. Gómez, P. D. Mininni, and P. Dmitruk, Adv. Space Res. 35, 899 (2005).

[52] D. O. Gómez, P. D. Mininni, and P. Dmitruk, Phys. Scr. 2005, 123 (2005).

[53] P. Mininni, D. Rosenberg, R. Reddy, and A. Pouquet, Parallel Computing 37, 316 (2011).

[54] C. Canuto, M. Hussaini, A. Quarteroni, and T. Zang, Spectral Methods in Fluid Dynamics, corrected 3rd print ed. (SpringerVerlag, Berlin/New York, 1988).

[55] G. Falkovich, Phys. Fluids 6, 1411 (1994).

[56] G. Krstulovic and M. Brachet, Phys. Rev. Lett. 106, 115303 (2011).

[57] B. Miquel and N. Mordant, Phys. Rev. E 84, 066607 (2011).

[58] M. S. Longuet-Higgins, J. Fluid Mech. 16, 138 (1963).

[59] E. Herbert, N. Mordant, and E. Falcon, Phys. Rev. Lett. 105, 144502 (2010).

[60] A. Azzalini, Scandinavian Journal of Statistic 12, 171 (1985).

[61] M. A. Tayfun, J. Geophys. Res.: Oceans 85, 1548 (1980).

[62] Y. Choi, Y. V. Lvov, and S. Nazarenko, Phys. Lett. A 332, 230 (2004).

[63] Y. V. Lvov and S. Nazarenko, Phys. Rev. E 69, 066608 (2004).

[64] Y. Choi, Y. V. Lvov, and S. Nazarenko, Physica D: Nonlinear Phenomena 201, 121 (2005). 